\title{
Nanoporous PMMA: A novel system with different acoustic properties
}

\author{
B. Notario ${ }^{a, *}$, A. Ballesteros ${ }^{a}$, J. Pinto ${ }^{b}$, M.A. Rodríguez-Pérez ${ }^{a}$ \\ a Cellular Materials Laboratory (CellMat), Condensed Matter Physics Department, University of Valladolid, 47011 Valladolid, Spain \\ ${ }^{\mathrm{b}}$ Nanophysics-Smart Materials Group, Istituto Italiano di Tecnologia (IIT), Via Morego 30, 16163 Genova, Italy
}

\section{A R T I C L E I N F O}

\section{Article history:}

Received 22 October 2015

Received in revised form

6 January 2016

Accepted 9 January 2016

Available online 11 January 2016

Keywords:

Nanoporous polymeric materials

Acoustic properties

Absorption

Transmission loss

\begin{abstract}
A B S T R A C T
The acoustic properties of closed cell nanoporous and microporous poly(methyl methacrylate) (PMMA) foams have been well characterized, showing that nanoporous PMMA exhibit a different absorption coefficient and transmission loss behavior in comparison with microporous PMMA. Experimental differences may be explained by the different wave propagation mechanism in the micro and nanoscale, which is determined by the confinement of both the gas (Knudsen regime) and the solid phases. These results place nanoporous materials as a new class of polymeric porous material with potential properties in the field of acoustics, especially in multifunctional systems requiring a certain degree of soundproofing.
\end{abstract}

(c) 2016 Elsevier B.V. All rights reserved.

\section{Introduction}

Currently one of the most exciting topics in porous material science is focused on the reduction of the pore size of porous material from the micrometer to the nanometer scale using technologies with the potential to be up-scaled. These new materials known as nanoporous foams (two-phase structures composed of a continuous solid phase and either continuous or discontinuous gaseous phase whose pore size is about or below 100$200 \mathrm{~nm}$ [1]) are very promising due to several improvements in their physical properties. For instance, B. Notario and coworkers [2] showed in poly(methyl methacrylate) (PMMA) based nanoporous materials that a pore size reduction allowed a significant reduction of the thermal conductivity due to the decrease of the heat conduction transfer through the gas phase (Knudsen effect) and due to an increment of the tortuosity of the solid matrix. Later the same authors [3] reported that the modulus of elasticity, the mechanical behavior at high strain rates as well as the shore hardness of nanoporous PMMA foams was clearly increased in comparison to microporous materials. A similar study of the mechanical behavior of nanoporous polyetherimide (PEI) was carried out by d. Miller et al. [4], finding that the tensile toughness and the strain to failure were increased by a factor of 2-3 when pore size is in the nanoscale. Moreover, impact resistance of nanoporous PEI foams presented a higher value than that of the microporous PEI. Furthermore, Zhou and coworkers [5] found a higher flexural modulus and strength in nanoporous PEI as compare to the solid.

\footnotetext{
* Corresponding autor. Tel.: + 34983423194.

E-mail address: belen.notario@fmc.uva.es (B. Notario).
}

This combination of properties makes these materials promising and appropriate for a significant no. of applications in different market sectors: construction, insulation, automotive, etc.

Due to all the aforementioned differences found in the physical properties between micro and nanoporous systems, this paper aims at. studying the acoustic behavior of closed pore microporous and nanoporous PMMA foams to find potential differences due to the significant modification of the morphology of the nanoporous system. Once again a significant effect owing to scaling was found both in absorption coefficient and transmission loss, opening the door to the development of new polymeric nanoporous materials with promising acoustic properties.

\section{Experimental section}

PMMA was kindly supplied by Arkema Company (France) in. the form of pellets. This polymer presents a glass transition temperature $\left(T_{g}\right)$ about $112{ }^{\circ} \mathrm{C}$ and a density $(\rho)$ of $1180 \mathrm{~kg} / \mathrm{m}^{3}$.

PMMA pellets were compression molded into precursors of $155 \mathrm{~mm} \times 75 \mathrm{~mm} \times 4 \mathrm{~mm}^{3}$ using a two-hot plates press, cut at the desired dimensions (radius $=29 \mathrm{~mm}$; thickness $=4 \mathrm{~mm}$ ), and used later for foaming [3].

Foaming experiments were performed following the solid state foaming process previously optimized (details elsewhere [3]), modifying the saturation pressure between 13 and $32 \mathrm{MPa}$ (Table 1). After depressurization samples were introduce in a thermostatic water bath in a controlled foaming step, intended to obtain samples with similar relative densities to better compare the differences in the acoustic properties between them.

Dense skin of foamed samples was removed using a polishing 
Table 1

Main characteristics of the porous materials studied.

\begin{tabular}{lllllll}
\hline Sample & $\begin{array}{l}\text { Pore } \\
\text { size } \\
(\Phi) \\
(\mathrm{nm})\end{array}$ & $\begin{array}{l}\text { Relative } \\
\text { density }\end{array}$ & $\begin{array}{l}\text { Absorption } \\
\text { main fre- } \\
\text { quency } \\
\text { peak }(\mathrm{Hz})\end{array}$ & $\begin{array}{l}\text { Absorption } \\
\text { peak } \\
\text { height }\end{array}$ & $\begin{array}{l}\text { Absorption } \\
\text { normalized } \\
\text { coefficient }\end{array}$ & $\begin{array}{l}\text { TL Main } \\
\text { frequency } \\
\text { peak }(\mathrm{Hz})\end{array}$ \\
\hline $\mathrm{M}-1$ & 11605 & 0.54 & 3873 & 0.21 & 0.12 & 3453 \\
$\mathrm{M}-2$ & 7365 & 0.46 & 4085 & 0.25 & 0.15 & $\mathrm{X}$ \\
$\mathrm{N}-1$ & 330 & 0.42 & 3926 & 0.21 & 0.09 & 5477 \\
$\mathrm{~N}-2$ & 205 & 0.41 & 4171 & 0.25 & 0.11 & $\mathrm{x}$ \\
\hline
\end{tabular}

machine (model LaboPOl2-LaboForce3, Struers). The presence of inhomogeneities or defects inside the porous materials was analyzed by X-Ray radiography. The cellular structure of porous samples was characterized by scanning electron microscopy (SEM) (FEI, Quanta $200 \mathrm{~g}$ ). The average pore size was determined with a specialized software based on ImageJ/ FIJI [6].

The acoustic behavior of porous systems was evaluated by measuring the absorption and the transmission coefficient (ratio between the absorbed and the incident energy, and ratio between the transmitted and the incident energy, respectively) in. the frequency range of $500-6000 \mathrm{~Hz}$. For this purpose the two-microphone (absorption [7]) or the four-microphone method (transmission [8]) (model Brüel \& Kjaer, 4206) was used, according to ASTM E1050 and ISO 10534-2:2002 [9]. The accuracy of the results was assured by performing six measurements for each material. The normalized absorption coefficient $(\tilde{\alpha})$ was evaluated according to [10]:

$\tilde{\alpha}=\frac{\int_{f_{1}}^{f_{2}} \alpha(f) d f}{f_{2}-f_{1}}$

Where $f_{1}$ and $f_{2}$ correspond to the extremal frequencies among which the study was conducted (i.e. $f_{1}=500 \mathrm{~Hz}$ and $f_{2}=6000 \mathrm{~Hz}$ ).

\section{Results and discussion}

The procedure to determine the acoustic behavior requires samples without internal structural defects. Thus an important step in this work was the choice of suitable samples by using X-ray radiography [11]. This approach allows us detecting these internal inhomogeneities without damaging the sample (Fig. 1).

All the samples manufactured in this work were analyzed using this approach, selecting only those ones with a homogenous structure for their acoustic characterization.
Representative SEM images of the porous structure are shown in Fig. 2. The study of the structure allows distinguishing two groups of foams: microporous (top part of Fig. 2) and nanoporous (bottom part of Fig. 2) PMMA. The entire systems exhibit closed pore morphologies due to the manufacturing conditions. This kind of porous structure is completely different from the typical porous structure of open pore materials used in acoustic insulation; nevertheless, these porous materials will allow us to analyze the effects of change in the scale.

Fig. 3 shows the average absorption acoustic response of microporous (left) and nanoporous (right) PMMA foams, illustrating well-defined sound absorption spectrums. Both systems present main absorption values of $0.21-0.25$ around $4000 \mathrm{~Hz}$ (Table 1), and exhibit an absorption coefficient normalized (Eq. 1) between $0.10-$ 0.15 . While microporous materials exhibit only one wide peak, nanoporous materials show two narrower peaks at different frequencies $(2100 \mathrm{~Hz}$ and $5700 \mathrm{~Hz})$. Furthermore, at high frequencies $(6000 \mathrm{~Hz})$ nanoporous PMMA do not absorb, unlike micro. Owing to the complexity of analyzing the acoustic properties of porous materials (due to their multiple forms of wave attenuation), nowadays there is a lack of theoretical and experimental studies able to elucidate the underlying mechanisms of these systems (and especially of the nanoporous ones). Nevertheless, because of the statements given by M. Ayud et al. [12] (acoustic waves propagates in. the air according to Knudsen regime in the nanoscale, gas exhibits different molecular motions in nanopores, gas and solid present different acoustic absorption mechanisms within the nanometer regime) and the different behavior found previously by B. Notario and coworkers [2,3] between microporous and nanoporous foams (confinement effect of polymeric macromolecules in. the nanoscale, increased tortuosity of nanoporous materials, Knudsen regime in nanopores), it was expected to obtain a different experimental acoustic behavior between materials whose pore size is in a different scale (as demonstrated in Fig. 3). However, further studies are needed to understand the real underlying phenomena.

Due to the low absorption coefficient value, these materials will be classified as low absorbers materials. However, although they present $\alpha$ values lower than the polyurethane (PU) ( $\alpha=0.97$ at $4000 \mathrm{~Hz}$ ), they exhibit $\alpha$ values well above than that of other materials commonly used in. construction like concrete $(\alpha=0.04$ at $4000 \mathrm{~Hz}$ ) or wood $(\alpha=0.07$ at $4000 \mathrm{~Hz}$ ). Thus they could be used in multifunctional systems requiring a certain degree of soundproofing as well as in improved thermal isolation or mechanical properties.

Transmission loss (TL) behavior of microporous (M-1) and

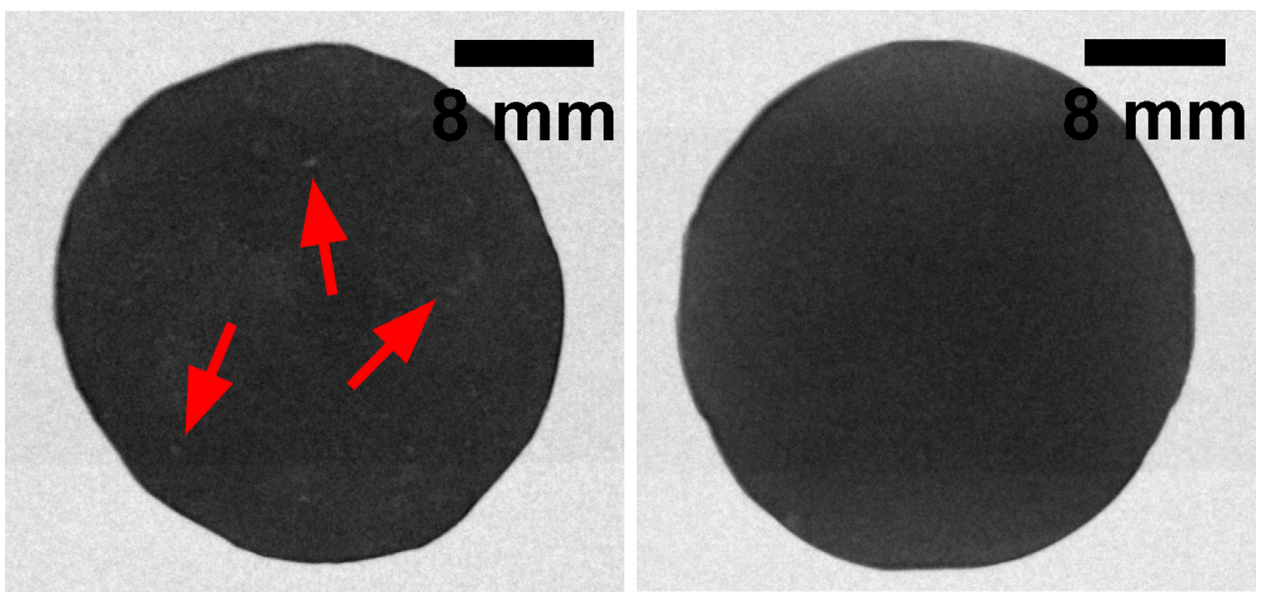

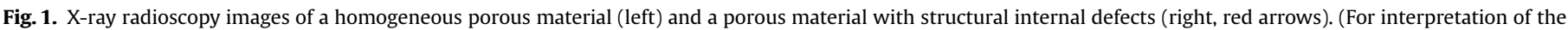
references to color in this figure legend, the reader is referred to the web version of this article.) 
a

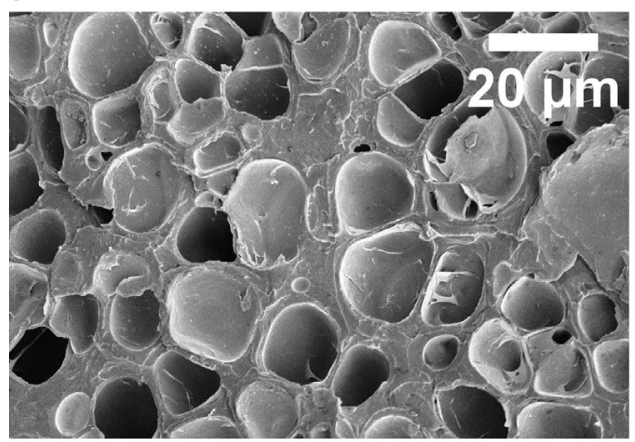

C

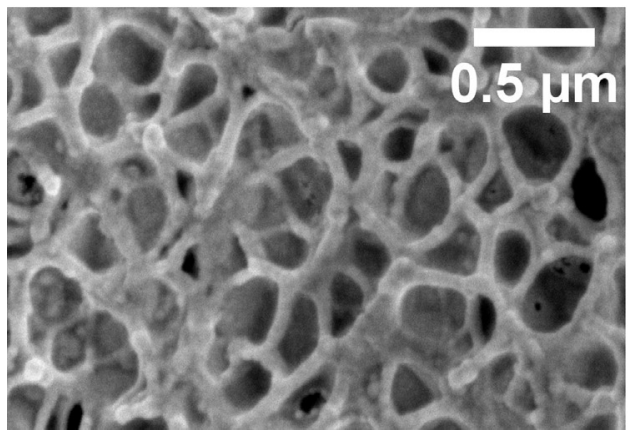

b

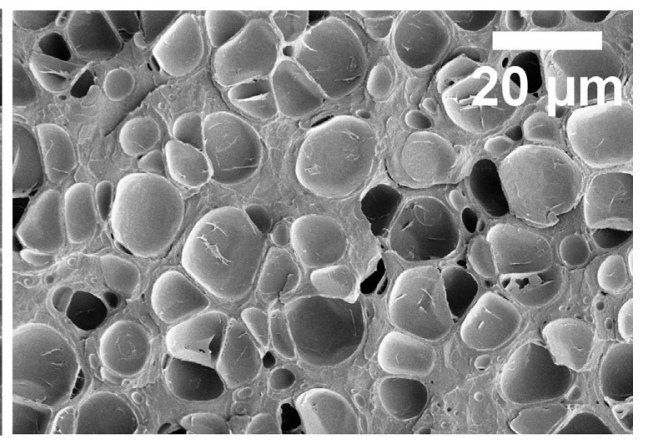

d

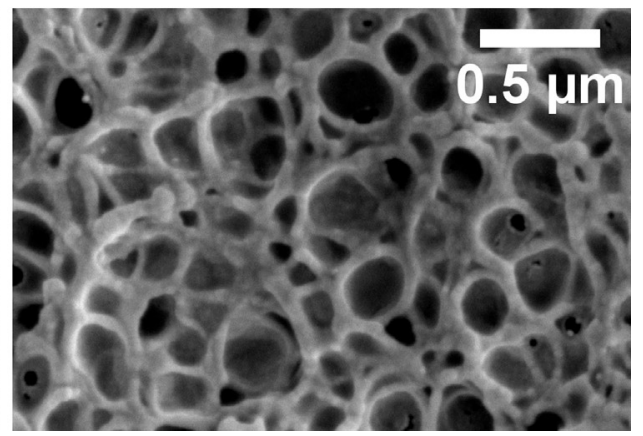

Fig. 2. SEM micrographs of the porous structure of (a) M-1 PMMA foam, (b) M-2 PMMA foam, (c) N-1 PMMA foam, (d) N-2 PMMA foam.
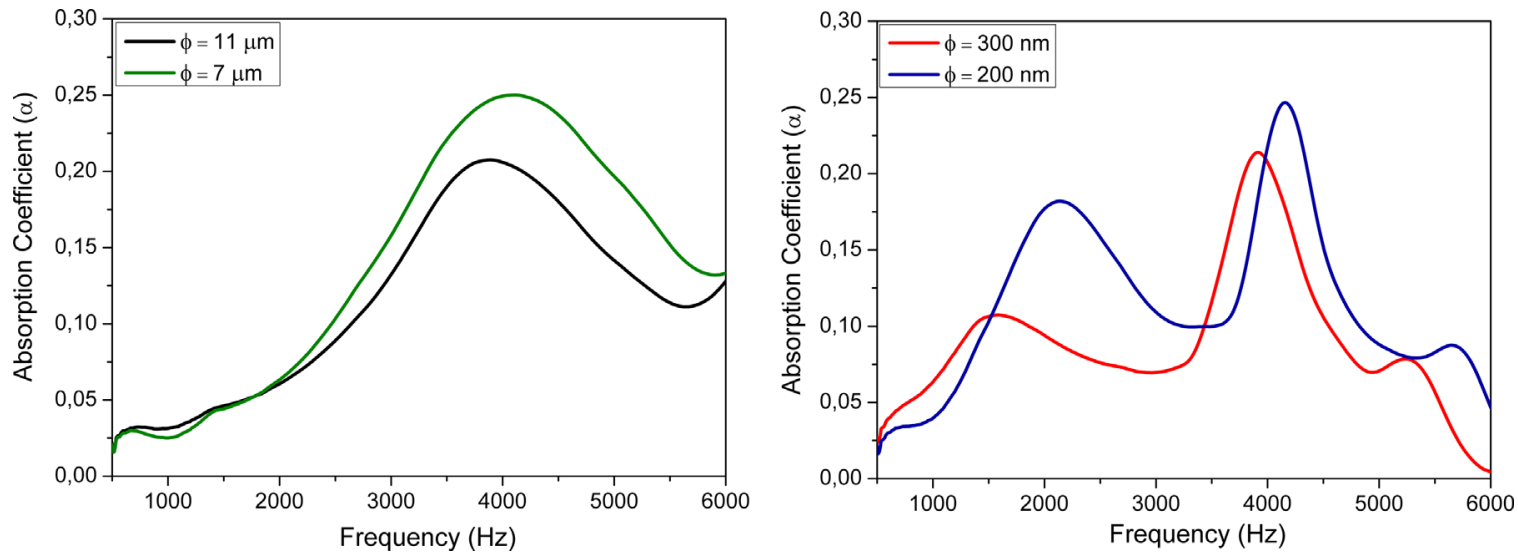

Fig. 3. Average absorption coefficient as a function of frequency. Left) Microporous PMMA Right) Nanoporous PMMA.

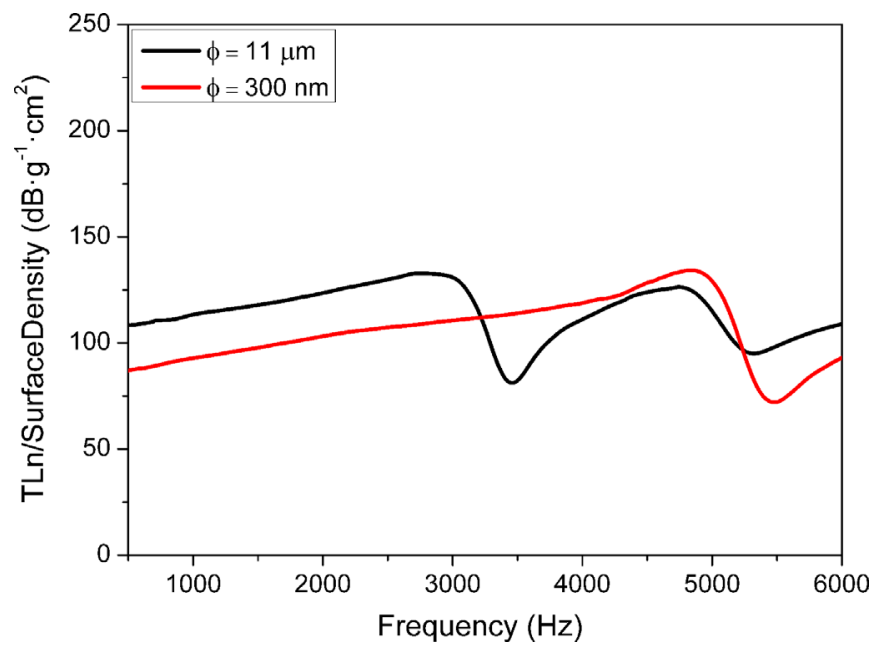

Fig. 4. Average TL coefficient divided by the surface density for a microporous and a nanoporous PMMA foams. nanoporous samples $(\mathrm{N}-1)$ was also analyzed. The average of the transmission loss spectrums are shown in Fig. 4. Again important differences can be observed in the acoustic behavior: nanoporous foams present one peak around $5500 \mathrm{~Hz}$, whereas microporous materials present two: around 3450 and $4500 \mathrm{~Hz}$ (Table 1). Again a scale change results in a different acoustic behavior, although further studies are needed to explain these differences.

\section{Conclusions}

in. this paper the acoustic properties of microporous and nanoporous PMMA foams were studied, finding a different acoustic behavior both in. the absorption coefficient and transmission loss. It is the first time that such systems are studied, and thus further studies are needed to understand the underlying mechanisms. These results are very promising, making nanoporous materials potential candidates in the sound field, especially in multifunction systems that require a certain degree of soundproofing. 


\section{Acknowledgments}

Financial support from FPI Grant BES-2013-062852 (B. Notario) from the Spanish Ministry of Education, MINECO (MAT 201234901), and the Junta of Castile and Leon (VA035U13) is gratefully acknowledged.

\section{References}

[1] L.J. Gibson, M.F. Ashby, Cellular Solids: Structure and Properties, 2nd ed. Cambridge University Press, Cambridge, 1997.

[2] B. Notario, J. Pinto, E. Solorzano, J.Ad Saja, M. Dumon, M.A. Rodriguez-Perez, Experimental validation of the Knudsen effect in nanocellular polymeric foams, Polymer 56 (2015) 57-67.

[3] B. Notario, J. Pinto, M.A. Rodriguez-Perez, Towards a new generation of polymeric foams: PMMA nanocellular foams with enhanced physical properties, Polymer 63 (2015) 116-126.

[4] D. Miller, V. Kumar, Microcellular and nanocellular solid-state polyetherimide
(PEI) foams using sub-critical carbon dioxide II. Tensile and impact properties, Polymer 52 (2011) 2910-2919.

[5] C. Zhou, N. Vaccaro, S.S. Sundarram, W. Li, Fabrication and characterization of polyetherimide nanofoams using supercritical $\mathrm{CO}_{2}$, J. Cell. Plast. 48 (2012) 239-255.

[6] M.D. Abràmoff, P.J. Magalhães, S.J. Ram, Image processing with ImageJ, Biophotonics Int. 11 (2004) 36-42.

[7] H. Koruk, An assessment of the performance of impedance tube method, Noise Control. Eng. J. 62 (2014) 264-274.

[8] J.G. Proakis, D.G. Manolakis Introduction to Digital Signal Processing. UK1988.

[9] ASTM. Standard Test Method for Impedance and Absorption of Acoustical Materials Using a Tube, Two Microphones and a Digital Frequency Analysis System.

[10] M.A. Rodriguez-Perez, M. Álvarez-Láinez, J.A. de Saja, Microstructure and physical properties of open-cell polyolefin foams, J. Appl. Polym. Sci. 114 (2009) 1176-1186.

[11] J. Escudero, E. Solorzano, M.A. Rodriguez-Perez, F. Garcia-Moreno, J.A. De Saja, Structural characterization and mechanical behaviour of ldpe structural foams. A comparison with conventional foams, Cell. Polym. 28 (2009) 289-302.

[12] M. Ayub, A.C. Zander, C.Q. Howard, B.S. Cazzolato, D.M. Huang A review of MD Simulations of Acoustic Absorption Mechanisms at the Nanoscale. Proceedings of Acoustics 2013. Australia2013. 